\title{
Nouvelles formules d'ordonnance pour la prescription des stupéfiants
}

\author{
C. Bodenschatz
}

Swissmedic met à disposition des médecins, à partir de janvier 2005, de nouvelles formules d'ordonnance pour la prescription des stupéfiants. A titre de nouveauté, celles-ci permettent les prescriptions multiples sur une seule ordonnance et vise à diminuer les contraintes administratives liées à la prescription de stupéfiants.

\section{Pratique de prescription actuelle}

Un stupéfiant par formule d'ordonnance Même en l'absence de contrainte légale sur ce point, la pratique veut que qu'un seul stupéfiant soit prescrit par ordonnance. Si le patient a besoin de deux stupéfiants ou plus, de même que plusieurs dosages ou formes pharmaceutiques, il se voit remettre une deuxième ou une troisième ordonnance.

\section{Nouvelle pratique}

\section{Plusieurs stupéfiants par formule} d'ordonnance

Pour limiter les contraintes administratives souvent déplorées, de nouveaux blocs d'ordonnances pour stupéfiants seront utilisables à partir de janvier 2005. Elles permettront de prescrire jusqu'à trois stupéfiants, ou des dosages ou formes pharmaceutiques différentes, par ordonnance. Pour éviter toute utilisation abusive, les lignes non utilisées devront impérativement être biffées. Un exemple de formule d'ordonnance correctement rempli est inclus dans le nouveau bloc.

\section{Autres points importants}

Correspondance:

Caroline Bodenschatz Swissmedic

Division stupéfiants

Erlachstrasse 8

CH-3000 Berne 9
Réglementation de la remise des différentes copies

Une note indiquant à qui remettre les copies de différentes couleurs figure désormais sur chaque formule. Il n'existe en effet pour l'heure pas de règle en la matière et la pratique n'est donc pas uniforme. Désormais, la copie bleue restera dans le bloc du médecin alors que les feuillets blanc et rose seront remis au patient pour l'obtention des stupéfiants à la pharmacie.

\section{Validité de la nouvelle ordonnance pour stupéfiants}

Aucun changement n'intervient en ce qui concerne la validité de l'ordonnance, ni les quantités prescrites. Pro memoria: la prescription de stupéfiants est valable au maximum un mois à compter de la date d'établissement de l'ordonnance, cette durée peut être prolongée de deux mois et doit être indiquée sur l'ordonnance.

\section{Commande des nouvelles formules d'ordonnance pour stupéfiants}

Pour passer commande, les médecins doivent, comme aujourd'hui, adresser aux autorités sanitaires cantonales compétentes la fiche de commande se trouvant dans le bloc.

\section{Validité des anciennes formules} d'ordonnance

Les actuelles ordonnances de stupéfiants restent valables. La différence majeure entre les deux versions réside dans le fait que les nouvelles ordonnances permettent de prescrire plusieurs stupéfiants sur une seule ordonnance, ce qui n'est pas possible dans la version actuelle.

\section{D'autres questions?}

Les autorités sanitaires cantonales compétentes, ainsi que Mme Caroline Bodenschatz (tél. 03132491 87, e-mail: caroline.bodenschatz@ swissmedic.ch) et Mme Isabella Saurer (tél. 031 32491 89. e-mail: isabella.saurer@swissmedic.ch), Division stupéfiants de Swissmedic, répondent volontiers à vos questions. 